\title{
Line shifts and sub-pc super-massive binary black holes
}

\author{
Saša Simić ${ }^{1}$ Luka Č. Popović ${ }^{2}$
}

\begin{abstract}
Here we discuss the possibility of supermassive binary black hole (SMBBH) detection, using the shifts of the broad lines emitted from a binary system. We perform a number of simulations of shapes and shifts of $H_{\beta}$ lines emitted from SMBBHs, taking into account the emission from two different regions located around both black holes, and kinematical effects which should be present in a SMBBH. In the model we connect the parameters of the lines with the mass of black holes and find that the peak shift depends, not only on kinematical effects of system rotation and black hole mass ratio, but it is also a function of the individual masses of the two black holes (BHs).
\end{abstract}

Keywords line shifts; binary black holes

\section{Introduction}

It is widely accepted that mergers play an important role in the galaxy formation and evolution, and mergers of supermassive black holes ( $\mathrm{SMBHs}$ ) are expected to be present at the centers of a number of galaxies (Begelman et al. 1980). In the case that a supermassive binary black hole (SMBBH) system is surrounded by gas, one can expect that observational effects may be present as a combination of the accretion onto one or both of the SMBHs and the dynamics of the binary system (see Popović 2012). In some cases, this could result in a binary Active Galactic Nucleus (AGN) system, that is similar to a single AGN, i.e. the system emits a broad band electromagnetic spectrum with

Saša Simić

Luka Č. Popović

Astronomical Observatory, Volgina 7, Belgrade

${ }^{1}$ Faculty of Science, Radoja Domanovica 12, Kragujevac, Serbia.

${ }^{2}$ Astronomical Observatory, Volgina 7, Belgrade, Serbia. broad and narrow emission lines. Due to the dynamics and composition of the two line emitting regions, however, the line profiles and shifts are expected to show some specific characteristics (see Popović 2012).

Based on the analysis of Very Large Telescope integral field spectroscopy and Hubble Space Telescope (HST) imaging SMBBHs are detected on a kpc scale (see e.g. Woo et al. 2013, 2014), but there is a problem to detect the sub-pc scale SMBBHs. It seems that sub-pc SMBBHs may be detected only using the spectral characteristics of a binary system (for a review, see Popović 2012; Bogdanović 2015).

The broad lines emitted from an AGN are used to probe of the geometry of the broad line region (BLR). The BLR seems to be close to the central $\mathrm{SMBH}$, since the reverberation technique gives the sizes of the BLR as an order of several light days to several light weeks (see Kaspi et al. 2005)). There is a group of AGNs that emit very broad and complex line profiles, which could be interpreted as two displaced peaks, one blueshifted and one redshifted from the systemic velocity defined by the narrow lines, or a single peak shifted line. It has been proposed that such line shapes could indicate a SMBBH system (see e.g. Gaskell 1983; Popović et al. 2000; Shen \& Loeb 2010; Tsalmantza et al. 2011; Eracleous et al. 2012; Popović 2012; Bon et al. 2012; Liu et al. 2014; Bogdanović 2015; Runnoe et al. 2015, etc.).

Here we discuss how the presence of a SMBBH would affect the shift of broad lines and what might be the observational consequences. First we describe our model of a SMBBH, and after that we analyze a number of simulations (combining different masses of components and different dynamical effects). The simulations have been performed to investigate line shift effects in broad lines due to emission from SMBBH. We specifically in- 
corporate some empirical relationships between the size of the gas, line intensity and $\mathrm{BH}$ masses in the model.

\subsection{SMBBH and broad line shift}

The idea that AGN activity is triggered by mergers is not new (see Komberg 1968a,b), however it is hard to spatially resolve (i.e. direct imaging) a binary system at sub-pc scale at the center of an AGN. Therefore, spectroscopy is a tool to study some effects of a SMBBH in the centers of AGNs. Spectroscopic studies may provide a method for identifying binary AGNs at sub-pc scale, through the search of velocity shifts in the complex broad line profile that is caused by orbiting the two BHs around common center of mass (see e.g. Gaskell 1983; Boroson \& Lauer 2009). However, it is interesting that of those AGNs, which have been detected as SMBBH candidates by spectroscopic methods, seem to be controversial because the line shifts and shapes may be explained by other phenomena in the BLR (as e.g. double-peaked lines with disc emission, or strong shift and asymmetry due to inflows/outflows Eracleous et al. 1997; Gaskell 2010) and/or there are some disagreements with other observational facts (e.g. long-term variability does not show expected the line profile variability Decarli et al. 2010a; Shapovalova et al. 2015).

First Gaskell (1983) discussed the shifted emission lines in light of the SMBBH hypothesis and reported about two quasars (see Fig. 1 and 2 in the paper of Gaskell 1983). The broad lines in the quasars $0945+076$ and $1404+285$ were off-centered (shifted with respect to the systemic velocity) to $-2100 \mathrm{~km} \mathrm{~s}^{-1}$ and $+2700 \mathrm{~km} \mathrm{~s}^{-1}$, respectively. The idea about SMBBHs in the center of AGNs has come into focus again nearly three decades later. There is a number of AGNs that show shifts in their broad emission lines and are good candidates for the SMBBH scenario (see Tsalmantza et al. 2011; Eracleous et al. 2012; Runnoe et al. 2015). In the framework of a $\mathrm{SMBBH}$ system there are some expected long term effects that apparently are not confirmed by observations. Let us mention here two interesting cases with large shift which may indicates SMBBH system. The first case is the AGN $4 \mathrm{C}+22.25$ where Decarli et al. (2010a) found that the $\mathrm{H} \beta$ and $\mathrm{H} \alpha$ lines show very broad line profiles (FWHM $\sim 12,000 \mathrm{~km} \mathrm{~s}^{-1}$ ), faint fluxes, and extreme offsets (due to the redshift) (8700土 $1300 \mathrm{~km} \mathrm{~s}^{-1}$ ) with respect to the narrow emission lines, but the line profiles do not vary in a period of 3.1 years (Decarli et al. 2010a). The second example is the quasar E1821+643 where the broad Balmer lines are redshifted by $\sim 1000 \mathrm{kms}^{-1}$ relative to the narrow lines and they have high red asymmetry Robinson 2010; Shapovalova et al. 2015). Recent monitoring of the same source (performed over an interval of 20 years), however, showed that there is no high variability in the line profile shapes and shifts, which do not agree with the predicted long-term variability (see Shapovalova et al. 2015). In spite of observational facts, one can expect that the line profiles in a long term period should vary (see Bon et al. 2012; Popović 2012).

We should mention here that the highly shifted broad emission lines may indicate two possible scenarios of a SMBBH: i) the case where in a SMBBH system only one component has a BLR, and ii) emission of the BLR bound to a recoiling SMBH, i.e., in the stage following coalescence. There are several AGNs with a relatively large shift of the broad line components (see e.g. Tsalmantza et al. 2011; Eracleous et al. 2012; Runnoe et al. 2015). Among them SDSS J092712.65+294344.0 was the first candidate for a recoiling SMBH (Komossa et al. 2008), with a broad component blueshift of $2650 \mathrm{~km} \mathrm{~s}^{-1}$ relative to the narrow emission lines. One can expect that both SMBH have emitting region. In this case there can be broad lines with specific shapes (see Bon et al. 2012), or lines with two peaks. Here we will discuss only the case where we have a binary system which has one or two broad line emitting regions. We will not consider recoiling black holes. Here we perform a number of models taking into account the dynamical and physical effects connected with the masses of black holes and their separations.

\section{Model}

In order to explore the shift of the total broad line emission, which is the composite of the emission from two BLRs located around the SMBH members of a binary system, we propose a relatively simple model, which takes into account the dynamics of the SMBBH similarly to Popović et al. (2000) and Shen \& Loeb (2010). Moreover, our model incorporates the properties of the BLRs around BHs in more detail; the broad emission line parameters are correlated with the masses of the central BHs, as revealed by studies involving reverberation techniques (Wu et al. 2004; Kaspi et al. 2005; Peterson 2014).

\subsection{Dynamics of SMBBH}

Basic theory of motion of binary systems is very well established. Here we consider the general case where 
two components with masses $m_{1}$ and $m_{2}$ and eccentricity $e_{1}$ and $e_{2}$ revolve around common the center of mass located at a common focus of the elliptical orbit of BHs. In such case the major axes of the ellipses are:

$$
a_{1}=\frac{R}{(1+q)\left(1+e_{1}\right)}
$$

and

$$
a_{2}=\frac{q R}{(1+q)\left(1+e_{2}\right)}
$$

where $R$ presents the maximum separation between components and $q$ denotes the component mass ratio $m_{1} / m_{2}$. At any particular moment during the orbital period one can find the position of the components with respect to the barycenter using the basic equation of the ellipse:

$$
r_{i}(t)=\frac{a_{i}\left(1-e_{i}^{2}\right)}{1-e_{i} \cos (\omega t)} .
$$

The period of rotation of such system $P_{\text {orb }}$ and the orbital velocities of components $v_{i}(t)$ are (see Paczynski 1971; Yu \& Lu 2001):

$$
\begin{aligned}
& P_{\text {orb }}=210\left(\frac{R_{S}}{0.1 \mathrm{pc}}\right)^{3 / 2}\left(\frac{2 \times 10^{8} M_{\odot}}{m_{1}+m_{2}}\right)^{1 / 2} \mathrm{yr} \\
& v_{i}(t)=1.5 \times 10^{3} \sqrt{\frac{0.1 \mathrm{pc}}{r(t)}} \sqrt{\frac{m_{1}+m_{2}}{2 \times 10^{8} M_{\odot}}}\left[\frac{2 m_{1} m_{2}}{m_{i}\left(m_{1}+m_{2}\right)}\right]
\end{aligned}
$$

with $i$ denoting the two components $(i=1,2), R_{S}$ mean component distance, $r_{i}(t)$ represents the $i$-th component position relative to the common focus as a function of time $t$. The parameter $\mu_{i}$ replace expression $\mu_{i}=\sin (\theta) \cos (\omega t+(i-1) \pi)$, where $\theta$ is the angle between the normal to the orbital plane and the lineof-sight of an observer (inclination of the orbit) and $\omega$ angular velocity of the system.

We assume that each component has its own accretion disc that represents a source of emission able to produce broad lines in the BLR.

\subsection{Physical properties of BLRs and broad line parameters}

In the standard AGN model a large region of moderate density $\left(n_{e} \sim 10^{9} \mathrm{~cm}^{-3}\right)$ surrounds the accretion disc, also known as the Broad Line Region - BLR (see e.g. Sulentic et al. 2000). We suppose that most of the BLR is distributed in same plane as accretion disc itself, with inner parts overlapping the accretion disc and spans a few tens of light days in diameter (Kaspi et al. 2005). The kinematics of the BLR can be very complex, but it is clear that the properties of the BLR depend on the parameters of the central black hole (Peterson 2014). Here we use some results obtained from reverberation to constrain the BLR parameters, taking into account the BHs masses in the binary system. We will consider the $\mathrm{H} \beta$ line, since the line has been widely used in black hole determination and in the monitoring campaigns. We also suppose, that other optical lines, such are $F e I I$ or $M g I I$, if they are generated by same BLR cloud, mimic the behaviour of the $\mathrm{H} \beta$ line, although similar analyse of their behaviour could give additional results for our computations. For the reason of simplicity we will suppose the Gaussian line profile instead of Lorentzian, since we are particulary interested in analyzing the line shifts which does not depends much on the line profile itself.

We should note here that the BLRs of two interacting $\mathrm{BHs}$ (within the small separation $(<1 p c)$ ) may be significantly affected by the dynamical interaction, and it may cause that the BLR kinematics in each BLR can be different that one observed in the case of the single central BH. To avoid it, we take that the BLRs are significantly smaller than the distance between two $\mathrm{BHs}$, and assume that the gravitation of a black hole is dominant for the local BLR. In that case we can apply the empirical relationships obtained from the case of single BH. However, there may be some gas around the $\mathrm{SMBBH}$ that can contribute to the line profile, that we neglected in this model, since only a detailed magnetohydrodynamical study can give the gas distribution around such complex system, that is out of scope of this work. Moreover, such a component probably will contribute to the center of line (expected smaller dispersion velocity since the emitting gas in this region is not too close to BHs as the local BLRs), and will have negligible influence on the total line shift.

\subsubsection{Estimation of the BLR sizes}

We estimate the BLR size by using the empirical formulas (Kaspi et al. 2005):

$$
\frac{R_{B L R}}{10 \text { lt-days }}=(2.21 \pm 0.21)\left[\frac{\lambda L_{\lambda}(5100 \AA)}{10^{44} \operatorname{ergs~s}^{-1}}\right]^{0.69 \pm 0.05},
$$

where $\lambda L_{\lambda}(5100 \AA)$ is the total disc luminosity at $5100 \AA$; for different wavelengths different BLR dimensions can be computed, e.g., utilizing formulas given by Kong et al. (2006). Here we calculate the continuum emission at $5100 \AA$ for each of the two accretion discs. Given empirical dependence Eq. 3 is 
derived for the case of the low and intermediate luminosity quasars, and we will restrict our simulations for this case. Further computations could be conducted in the same way on the high-luminosity/high redshift quasars using the empirical relations presented in the Kaspi et al. (2007).

The disc has the inner radius $\rho_{\text {in }} \sim$ several $R_{g}$ and outer radius $\rho_{\text {out }} \sim R_{R L}$ ( $R_{g}$ is gravitational radius for each black hole separately given by $R_{g}^{i}=\frac{G m_{i}}{c}$ ). The outer radius can be estimated as the radius of the Roche lobe of two bodies (see Eggleton 1983)

$$
R_{R L}(x)=\frac{0.49 R_{s} x^{2 / 3}}{\left.0.6 x^{2 / 3}+\log \left[1+x^{1 / 3}\right]\right)},
$$

where $x$ is defined by the mass ratio of the components as follows: $x=q$ for the more massive $\mathrm{BH}$ and $x=1 / q$ for the less massive one. The inner radius of the disc is not exactly equal to the one $R_{g}$ since the radiation pressure is huge within one $R_{g}$, producing impossible conditions for any material to condensate and undergo laminar motion. This effect is known as AdvectionDominated Accretion Flow (ADAF) and it is present in the case of the spinning black holes described with the Kerr metric (see Narayan \& Yi 1994; Manmoto 2010). For this reason we adopted that $\rho_{i n}^{i}=3.5 R_{g}^{i}$ as inner radius of the disc. On the other hand the outer disc radius is just a half of the corresponding Roche radius since the disc can not entirely fill the space defined by $R_{R L}$ (see Yan et al. 2014).

The distribution of temperature over the radius of the disc follows the power law which in simplified form is given as $T_{\text {eff }}(\rho) \sim \rho^{-3 / 4}$ Shakura \& Sunyaev 1973). The emission model is assumed to be thermal in nature and the amount of radiation generated by other mechanisms (inverse Compton or synchrotron) is thought to be negligible for optical and UV bands. In order to compute the total amount of radiation coming from the surface of the disc one needs to integrate emission of the entire disc area. This allows the computation of the specific luminosity $L_{\lambda}(5100 \AA)$ that is used in Eq. 3. Consequently, we connect the size of the BLR with the individual masses of the two BHs and also with their mass ratio.

\subsubsection{The intensity of the $H \beta$ line}

The $\mathrm{H} \beta$ line intensity is estimated using empirical relation given by $\mathrm{Wu}$ et al. (2004):

$\log R_{B L R}($ lt-days $)=1.381+0.684 \cdot \log \left(\frac{\lambda L(H \beta)}{10^{42} \text { ergs s}^{-1}}\right)$.
We define $\lambda L\left(H_{\beta}\right) \equiv I_{\lambda 0}^{H \beta}$, the luminosity of the $H_{\beta}$ line, and substitute the $R_{B L R}$ with its coresponding expression from Eq. 4. This allow us to identify the individual contributions of the two components to the total line emission in the binary system.

\subsubsection{The width and shift of $H \beta$}

The $\mathrm{H} \beta$ line width is supposed to be proportional to the black hole mass, since more massive BHs induce higher velocities in the BLR (see Peterson 2014).

The velocity distribution in the BLR can be estimated based on the viral theorem as:

$$
v_{B L R}\left(m_{i}\right)=\sqrt{\frac{G m_{i}}{R_{B L R}}},
$$

giving the spectral line width dispersion as $\sigma_{i}=$ $\lambda_{H \beta} \frac{v_{B L R}\left(m_{i}\right)}{c}$. Note here that the velocity of gas in the BLR is a direct function of the associated BH mass and it is not directly tied into the mass ratio $q$. This will be very important in computing line shifts, since different mass combinations can produce the same mass ratio, but different orbital velocities of binary components, consequently different line shifts and velocity dispersion.

The line shift caused by gravitational effects in the close vicinity of the $\mathrm{BH}$ can be computed by $\Delta \lambda_{g}^{i}=$ $\frac{G m_{i}}{c R_{B L R}}$. For the BLR sizes adopted in our computation, however, the produced line-shift $\Delta \lambda_{g}^{i}$ is small compared to the shifts produced by the radial velocities of binary components. Consequently, one can neglect the effect of gravitational line shift from the line shape computation. On the other hand, one should include the shift of lines emitted from different components taking into account the dynamics of the system. We take that rest wavelength of lines is shifted according to

$$
\lambda=\lambda_{0}\left(1+z_{\text {dopp }}^{i}\right)
$$

where $z_{\text {dopp }}^{i}$ represents the ratio $v_{r}^{i} / c$, particulary for each component of the binary system.

\subsubsection{The line shapes}

We calculate line shapes of different configurations of SMBBH system as:

$$
I_{\text {tot }}(\lambda)=I_{1}(\lambda)+I_{2}(\lambda)
$$

where we assume that each component emits Gaussian line profile:

$$
I_{i}(\lambda)=I_{i}\left(\lambda_{0}\right) \exp \left[-\left(\frac{\lambda-\lambda_{0} \cdot\left(1+z_{\text {dopp }}^{i}\right)}{\sqrt{2} \sigma_{i}}\right)^{2}\right] \cos (\theta)
$$


Table 1 Computed values for duration of orbital phases $t$ [years] and corresponding component separations $r_{\text {sepp }}$ for

with $i$ designating the components 1 and $2, \lambda_{0}$ wavelength of $\mathrm{H} \beta$ line in laboratory frame, and $z_{\text {dopp }}^{i}$ Doppler corection for radial component velocities. We also ascribe a flat distribution to the BLR, coplanar with the orbital planes of the SMBBHs and therefore defined by the same inclination angle $\theta$.

\subsubsection{Model constraints and measured line parameters}

First of all, using this model, one has to adopt some constraints: a) since we derive the radius of the BLR from the continuum luminosity, one should check if the size of the BLR fit within the separation between the two $\mathrm{BH}$ components. In order to avoid semi-detached system we constrain our model that $R_{B L R 1}+R_{B L R 2}<R$. In first approximation, even if there are small interactions of BLRs, it should not had much influence on the dynamics of binary system, as well as on the emitted amount of radiation, since the interacting volumes are expected to be quite small in comparison with the whole region of BLRs. After several tests, we obtain that the limiting value of $R$ in considered models is $0.05 \mathrm{pc}$ for masses of the order of $10^{8} M_{\odot}$. b) As we investigate the role of $\mathrm{BH}$ mass on the line shapes and shifts, we explore the range of masses $10^{8}-10^{9} M_{\odot}$ for the two BHs. c) The orbital parameters as well as the mass ratio affect the total line shift (see Popović et al. 2000; Shen \& Loeb 2010). To explore individual mass effect we consider the cases $q=0.25,0.5$ and 1 , and we assume eccentricities $e_{1,2}=e_{1}=e_{2}=0.7$.

We would like to get time-scale estimates for an orbital period, which can be used to predict the longterm effects on line-shifts to compare against long-term monitoring campaigns for SMBBH candidates. Thus, we calculate the time for different phases (specific snapshots) and the corresponding separations between BHs, testing various mass ratios and maximum separations between the components.

As one can see from Table 1] the changes in the line shift (caused by dynamical effects) can be present in a time interval of the order of decades. The calculation for periods and distances has been performed for a SMBBH system with mass of the smaller component equal to $10^{9} M_{\odot}$ and a distance between components of $R=0.1$ pc.

To explore the shift in SMBBHs and its usage for detection of binary black holes we measure the shift of the peak (the maximum of the line). Additionally, we compute the wavelength of the line centroid and appropriate shift as:

$$
\lambda_{c}=\frac{\int I_{t o t}(\lambda) \lambda d \lambda}{\int I_{t o t}(\lambda) d \lambda}
$$

different dynamical parameters $q$. The eccentricity of the orbits is taken as $e_{1,2}=e_{1}=e_{2}=0.7$.

\begin{tabular}{|c|c|c|c|c|}
\hline parameters & $P_{\text {orb }} / 4$ & $P_{\text {orb }} / 2$ & $3 P_{\text {orb }} / 4$ & $P_{\text {orb }}$ \\
\hline $\mathrm{q}=1$ & 14.2 & 28.6 & 42.8 & 57.1 \\
$\mathrm{q}=0.5$ & 11.6 & 23.3 & 35.1 & 46.6 \\
$\mathrm{q}=0.25$ & 9.1 & 18.0 & 27.1 & 36.1 \\
\hline \hline$r_{\text {sepp }}(\mathrm{pc})$ & 0.03 & 0.017 & 0.03 & 0.1 \\
\hline
\end{tabular}

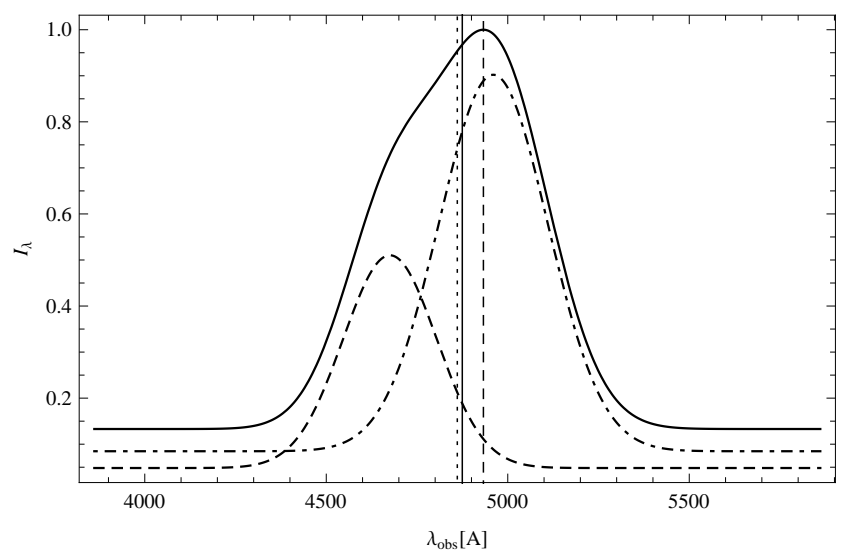

Fig. $1 \mathrm{H} \beta$ line for a $\mathrm{BBH}$ system, with mass ratio $q=0.5$ and particular component masses of $m_{1}=4 \cdot 10^{8}$ and $m_{2}=8 \cdot 10^{8} M_{\odot}$. Solid curved line shows a total $\mathrm{H} \beta$ line from component 1 (dashed curved line) and component 2 (dotdashed curved line). Vertical straight lines designate unperturbed wavelength (dotted line), shift of the peak (dashed line) and centroid (solid line) of combined $\mathrm{H} \beta$ line. Note: Centroid shift is intentionally slightly displaced from it's computed position for the reason of clarity.

$$
\delta \lambda_{c}=\lambda_{0}-\lambda_{c}
$$

The total line shape of the $\mathrm{H} \beta$ line generated by the sub-pc binary system of SMBHs (with masses of $m_{1}=$ $4 \cdot 10^{8}$ and $\left.m_{2}=8 \cdot 10^{8} M_{\odot}\right)$ and parameters $q=0.5$ and $R=0.05 \mathrm{pc}$ is presented in Fig. 1. As we can see in this example, the line shows a complex profile with a high blue asymmetry, showing a blue shoulder and a redshifted peak. We present the line peak and shift of the centroid with vertical lines. In this particular case the shift of the line peak is significantly larger than the shift of the line centroid.

\section{Results and discussion}

In previous sections we assume that both components of binary system retain their accretion discs and surrounding BLRs. However, it is of particular interest to investigate a system where just the smaller component 
has BLR, since in that case there is no line combination with the other component, and the resulting shift would be more clearly defined. For that reason we will discuss two cases in this section: $i$ ) both components have a BLR and $i i$ ) only the smaller component has a BLR.

\subsection{The case of both black holes with BLRs}

In the case of bound BHs orbiting around the common barycenter, their masses determine the dynamics of orbital motion and consequently the shifts of the components that contributing to the shift of the observed (total) line profile. As we noted above, we have compute the shape of the $\mathrm{H} \beta$ line for different combinations of masses and mass ratios $q$. We first consider the case of $q=1,0.5,0.25$, with associated individual $\mathrm{BH}$ masses of $m_{1}=1 \cdot 10^{8} M_{\odot}$ and $m_{2}=1,2,4 \cdot 10^{8} M_{\odot}$ (see Fig. 2 panels a), b), c)). We than change the individual $\mathrm{BH}$ masses to $m_{1}=1 \cdot 10^{9} M_{\odot}$ and $m_{2}=1,2,4 \cdot 10^{9} M_{\odot}$ while keeping the same $q$ ratios as before (see Fig. 2 panels $d), e), f)$ ). In both cases computations here refers to the quite specific configuration with longitudinal alignment between line-of-sight (LOS) and the axes of the elliptical paths of two BHs, for the reason that in such case radial velocities over the LOS are highest for the orbital phase $P_{\text {orb }} / 2$ and given inclination angle $\theta$, with the components at closest separation (see Table 11). Consequently a larger line shift during the orbit is expected.

As one can see in Fig 2, when increasing the mass of the second component $m_{2}$, both the intensity and the width of the associated line go up. Although the mass ratio is the same (panels $a-d, b-c, c-f$ ), both the shift and asymmetry of the total/composite line become more prominent in the more massive binary system (panels $d$-e-f compared to $a-b-c$ ). It shows, that beside the dynamical parameters (the mass ratio, phase and separations), the masses of components have an important influence on the line profiles and determine the detectability of the effect in SMBBH system. In Table 2 we give measured values of the line shifts for components (Columns 2 and 3), the total shifts (Column 4) and centroid shift (Column 5). As it can be seen, with bigger mass of the components the shift stays larger, and consequently the effect of a binary system is more pronounced.

With increasing the orbital eccentricity of BHs line shifts could be more defined in some particular phases of the orbit with minimum separation between components. For that reason we compute the shape of the total $\mathrm{H} \beta$ spectral line for different phases, see Fig. 3 In upper/top panels we present the case of low mass components with masses $m_{1}=4 \cdot 10^{8} M_{\odot}, m_{2}=8 \cdot 10^{8} M_{\odot}$ and $R=0.05 \mathrm{pc}$. The lower/bottom panels show the larger mass case with $m_{1}=4 \cdot 10^{9} M_{\odot}, m_{2}=8 \cdot 10^{9} M_{\odot}$ and $R=0.1 \mathrm{pc}$. In both considered cases with increasing the masses of the BHs, the BLR diameter also increase, so we intentionally increase separation $R$ to avoid mutual interaction of BLRs. As it is well known (see Popović et al. 2000; Shen \& Loeb 2010) during an orbital cycle, the broad line profile been changes, the asymmetry and shift acquiring different values. However, the asymmetry and line profile changes are expected to be more important in the case where the components have bigger masses $\left(\sim 10^{9} M_{\odot}\right)$. The most interesting is that only in one case (panel $e$ ) for higher mass case) there is clearly seen a double-peak profile that may indicate the emission of two BLRs. These simulations suggest that the asymmetric profiles are far more prevalent then the extreme double-peak ones.

Note here that different geometries could be applied in order to explain the observed broad spectral lines components. Double peaked lines could be a result of binary system dynamics, but it seems that double-peaked line shapes could be achieved when a single AGN has two or more broad line emitting regions, and if the emission of an accretion disc is dominant Eracleous et al. 1997; Popović 2004; Bon et al. 2009; Popović et al. 2011). In the case that a SMBBH system is emitting double-peaked line profiles, the distances between peaks has to be changed, and also, single peaked asymmetric profile can be observed in a monitoring campaigns, while in the case of doublepeaked profiles emitted by the disk one cannot expect a big changes in the shift of the peaks (Eracleous et al. 1997). Additionally, double peaked profiles originated by the disk-like region usually have a boost in the blue peak, and extended red wing (Eracleous et al. 1997; Popović et al. 2011), that is not a rule in the profiles of SMBBHs. The most important difference between double peaked lines from the disk and SMBBHs is that the line profiles of SMBBHs are changing dramatically during time (from single to double peaked), that is not case for the lines emitted from the disk (see the case 3C390.3 in Popović et al. 2011).

$W$ e should note here that observed broad line profiles in AGN can be complex and specific, as we mentioned above, the double-peaked profiles can be observed in a group of AGN (around 10\% of AGN, see Strateva et al. 2003; Eracleous \& Halpern 1994), which seems to be emitted from a disc-like geometry. On the other side, it seems that we can divided the AGN using the width of their profiles (as e.q. FWHM $>4000 \mathrm{~km} \mathrm{~s}^{-1}$, see Sulentic et al. 2009; Zamfir et al. 2010; Marziani et al. 2010) where broader lines show a red/blue asymmetry. Comparing 


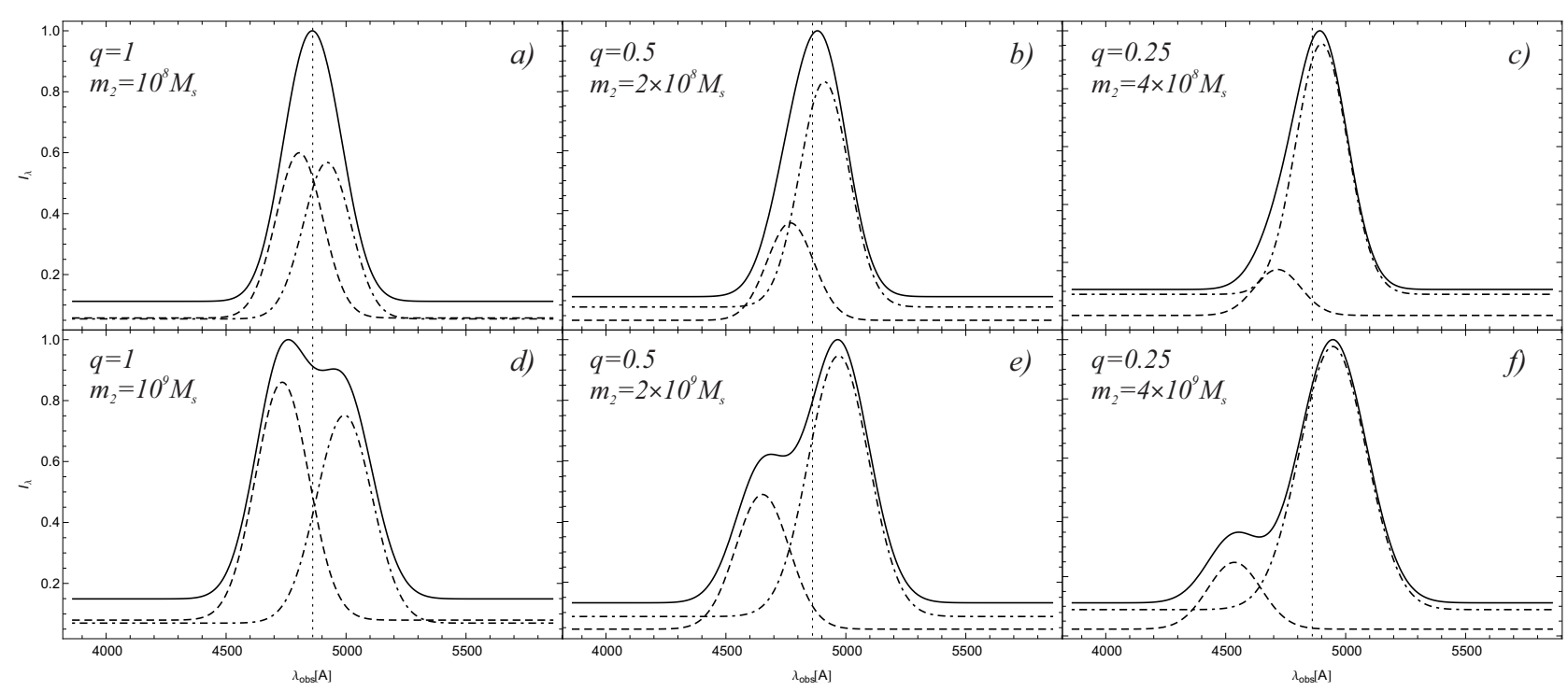

Fig. $2 \mathrm{H} \beta$ line shifts for different mass ratio $q$ and mass of components (given on plots). In first row (panels $a$ ), $b$ ), $c$ )) the maximum $\mathrm{BH}$ component separation is $R=0.05 \mathrm{pc}$, while in the second raw (panels $d$ ), e) and $f$ )) the maximum separation is $R=0.1 \mathrm{pc}$.
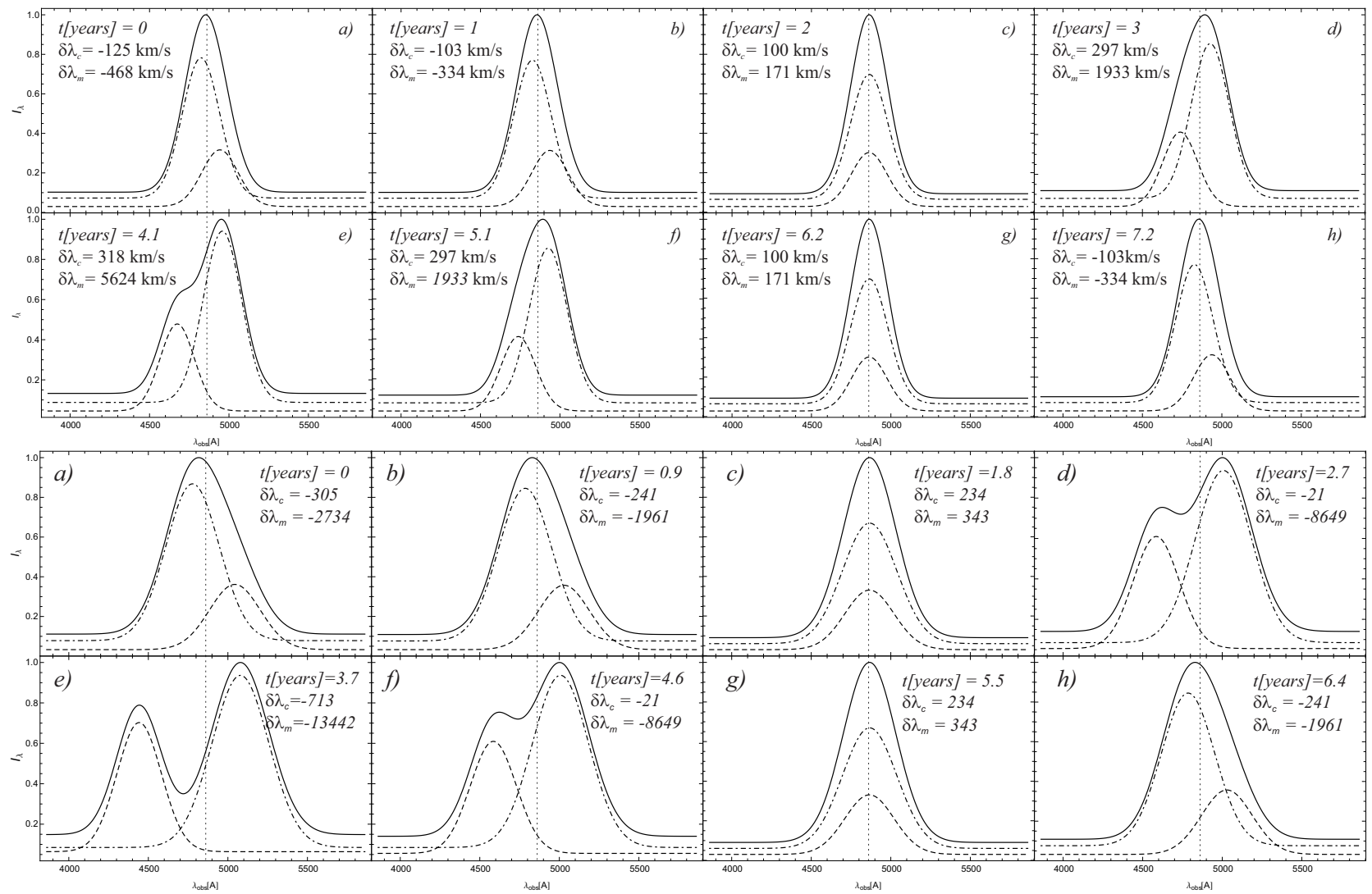

Fig. 3 The total $\mathrm{H} \beta$ line profiles during full orbits for two SMBBH systems. Upper panels (from $a$ ) to $h$ ) are given for the mass $m_{1}=4 \cdot 10^{8} \mathrm{M}_{\odot}$ and $R=0.05 \mathrm{pc}$, and lower panels (from $a$ ) to $h$ ) for $m_{1}=4 \cdot 10^{9} \mathrm{M}_{\odot}$ and $R=0.1 \mathrm{pc}$. For both systems we fixed $q=0.5$ and $e_{1}=e_{2}=0.7$. Dashed and dot-dashed lines represents contributions of the components to the total $\mathrm{H} \beta$ lines (solid lines). In the panels from $a$ ) to $h$ ) we indicate the corresponding phase $t$ in years, line centroid shift $\delta \lambda_{c}$ and peak shift $\delta \lambda_{m}$ in $\mathrm{km} / \mathrm{s}$ on each plot. 
Table 2 Numerical values of shifts presented in Fig. 2, First Column denotes the corresponding panel in Fig. 2] second and third Columns the shift of peaks for components, respectively. In fourth and fifth columns are given shifts of peak and centroid of the total line profile, respectively.

\begin{tabular}{|c|c|c|c|c|}
\hline panel & $\delta \lambda_{m}^{1}[\mathrm{~km} / \mathrm{s}]$ & $\delta \lambda_{m}^{2}[\mathrm{~km} / \mathrm{s}]$ & $\delta \lambda_{m}[\mathrm{~km} / \mathrm{s}]$ & $\delta \lambda_{c}[\mathrm{~km} / \mathrm{s}]$ \\
\hline$a)$ & -3450 & 3696 & -110 & 32 \\
$b)$ & -5711 & 3050 & 1266 & 278 \\
c) & -8915 & 2405 & 1935 & 451 \\
d) & -7828 & 8156 & -6268 & -110 \\
e) & -12881 & 6718 & 6482 & 333 \\
$f)$ & -20042 & 5294 & 5282 & 630 \\
\hline
\end{tabular}
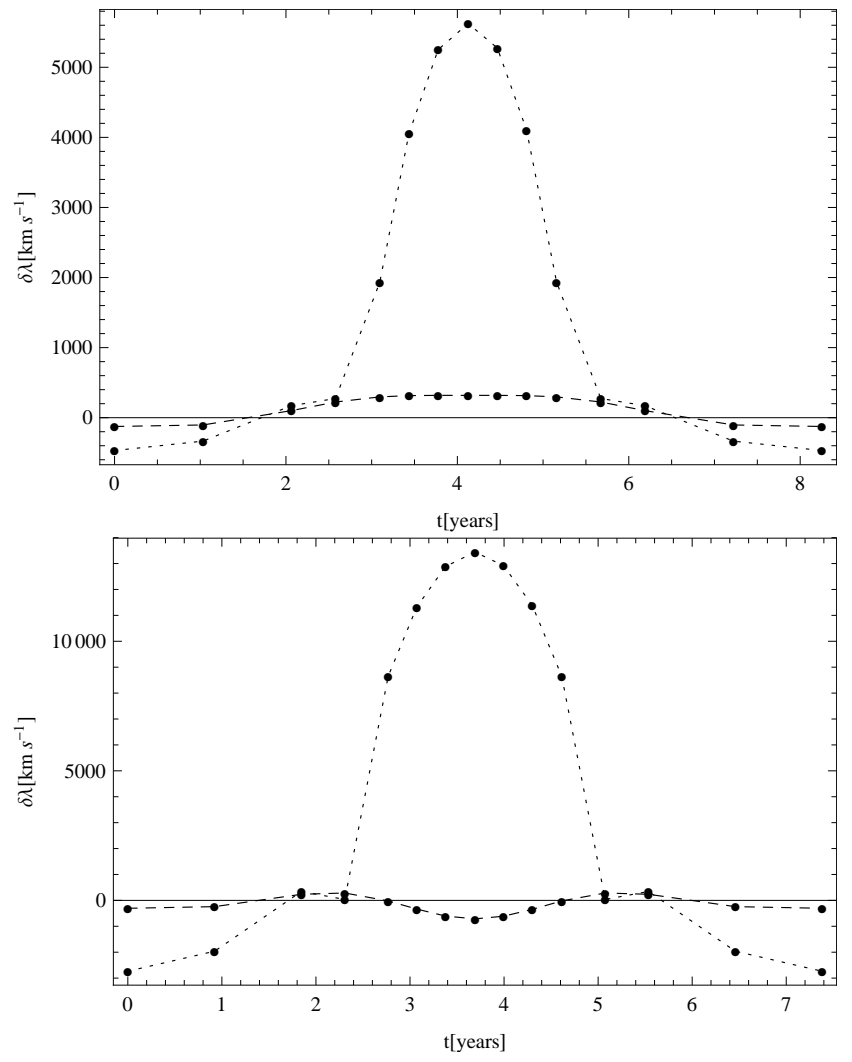

Fig. 4 The corresponding shifts (doted line) and line centroid shifts (dashed line) for the cases given in Fig. 3 Upper panel corresponds to the case of lower masses of components (upper panels in Fig. 3). our, modeled broad line profiles with observations we found that (see Fig. 3) all type of line profiles in the case of a $\mathrm{SMBBH}$ is expected. If there is a $\mathrm{SMBBH}$ with smaller masses, we can expect relatively narrow and symmetric broad line profiles during $2 / 3$ of the orbiting period, in the case of a massive SMBBH, the line profiles are mostly asymmetric. Therefore, to find more evidence about the structure of emitting system (SMBBH vs. complex BLR), a long-term monitoring, and analysis of the line profile (shift, width and intensity) variation is needed.

The time evolution of the total line peak shift and line centroid shift for the two cases shown in Fig. 3 are presented in Fig. 4. It is obvious that the peak shift is more prominent than the centroid shift. However it is interesting that only in a relatively short time-window (several years, see Fig. 4), the line shift is significant and could be easily measured, whereas, for about $3 / 4$ of a period the shift has small values.

$W$ e should note here, that some previous studies of a large number broad line AGN seem to suggest that blue-shifted peaks are more prevalent than redshifted ones (see e.g. Zamfir et al. 2010; Marziani et al. 2013) that cannot be explained by SMBBH hypothesis. This also supports the fact that the long term observations, which can indicate the changes in the line shift, may be used for detection of a SMBBH system.

\subsection{SMBBHs with one BLR}

For a compact binary system there is a possibility that just one BH has the BLR (see e.g. Armitage \& Nataraian 2002; Havasaki et al. 2007; Cuadra et al. 2009; Lodato et al. 2009; Popović 2012).

Good examples of subparsec BBH quasar are PG 1302-102 reported by Graham et al. (2015) and Mrk 231, see Yan et al. (2015), recently suggested via either periodical variations or peculiar continuum spectrum. PG 1302-102 is described as a binary SMBH pair with a total virial mass of $10^{8.5} M_{\odot}$, with observed period limiting the separation between the pair to 0.01 
pc. This means that the system has evolved well into the final parsec scale, with two component significantly different in their mass, as it can be concluded from fitting the spectral lines of Hydrogen series (see their Fig. 4). Also, Mrk 231 binary is high mass ratio system $(q \sim 50)$, with unique features in the optical-to-UV spectrum and the intrinsic X-ray weakness. In both cases the emission from the small BH component dominates and the disk associated with the big $\mathrm{BH}$ component emits little ionizing photons.

In such case the observed $\mathrm{H} \beta$ line is a singlet, which could be shifted around the rest wavelength. It is of particular interest to analyze the case when a component with bigger mass is without BLR. This is more probable scenario since there is a bigger chance that smaller mass component retain it's accretion disc and BLR.

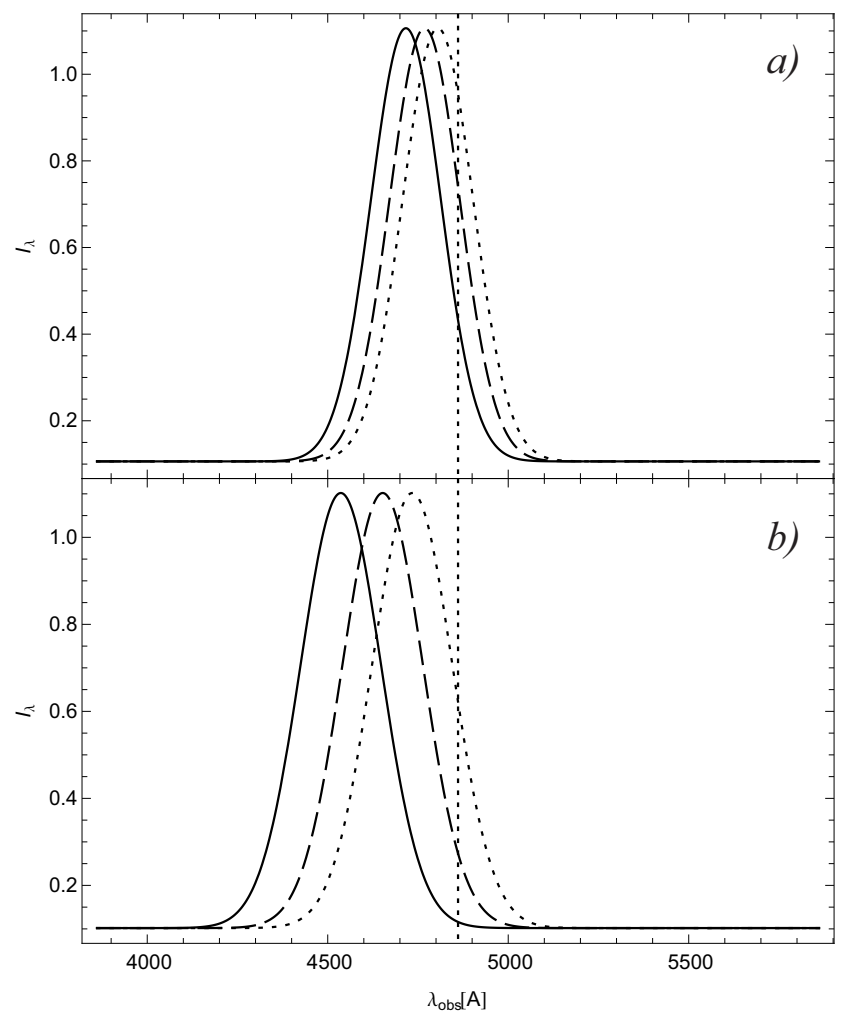

Fig. 5 The $\mathrm{H} \beta$ line from a single BLR associated with the lower mass BH component of binary system. Panel $a$ ) presents the computation for parameter $R=0.05 \mathrm{pc}$ and the mass of the bigger component having values $m_{2}=1,2,4$. $10^{8} M_{\odot}$. Panel $b$ ) shows the case of $R=0.1 \mathrm{pc}$ and $m_{2}=$ $1,2,4 \cdot 10^{9} M_{\odot}$. Used line designations are: dotted line $q=1$, dashed line $q=0.5$, and solid line $q=0.25$.

As we can see in Fig. 5] and Table 3 where we present the computations for the case of just a lower mass $\mathrm{BH}$ containing it's BLR, the total shift of the $\mathrm{H} \beta$ line is significantly greater than the shift in the case of the combined line produced by two BLRs.
Table 3 Centroid shift for single $\mathrm{H} \beta$ line presented in Fig. 5. The first three rows of data are for the case when $m_{2}=$ $1,2,4 \cdot 10^{8} M_{\odot}$, while last three rows are for $m_{2}=1,2,4$. $10^{9} M_{\odot}$.

\begin{tabular}{|c|c|}
\hline$q$ & $\delta \lambda_{1}^{c}[\mathrm{~km} / \mathrm{s}]$ \\
\hline 1 & -1844 \\
0.5 & -3047 \\
0.25 & -4745 \\
\hline 1 & -4517 \\
0.5 & -7440 \\
0.25 & -11578 \\
\hline
\end{tabular}

Also, in this case, we obtain that shifts in the single component depends on the mass, as expected. However, if we have the same mass ratios, one can expect that the shift will be larger in the higher mass system. As one can see in Table 3, when the mass is increased by an order of magnitude, the shift increases 2-3 times.

Line shifts in the single BLR case is caused by the orbital motion of the smaller component. Our computation in Fig. 5 is performed for the highest radial velocity. As we can see in Fig. 5, and Table 3, for both considered cases (a) and b)), the shift is, approximately, a linear function of the mass, i.e. $\delta \lambda_{1}^{c} \sim m_{2}$. This is in accordance with expectations since with increase of $m_{2}$, the center of mass of the system is closer to bigger component, and smaller component will have higher radial velocity, producing higher line shifts.

In Fig. 6] we show the time-evolution for the line centroid and shift over a full orbital period of the rather peculiar system we simulate. We note that the line is very symmetric at all phases, so the line asymmetry as shown in the case of two emitting BLRs, cannot be seen. A system like this cannot account for highly asymmetric lines that show complex profiles.

\section{Conclusion}

In this paper we consider the possibility to detect the line shift of SMBBH systems. To investigate the shift effect we develop a model, which is taking into account the dynamical effects similar to Popović et al. (2000) and Shen \& Loeb (2010), but in contrast with models given in these papers, we include physical properties of the emitting regions in both components of a $\mathrm{SMBBH}$ system. We constrain the radius of the BLR taking into account the emission of the continuum at $5100 \AA$ (estimated from disc emission around components), the intensity of the $\mathrm{H} \beta$ line of each component considering empirical relationship between the $\mathrm{H} \beta$ line intensity and 


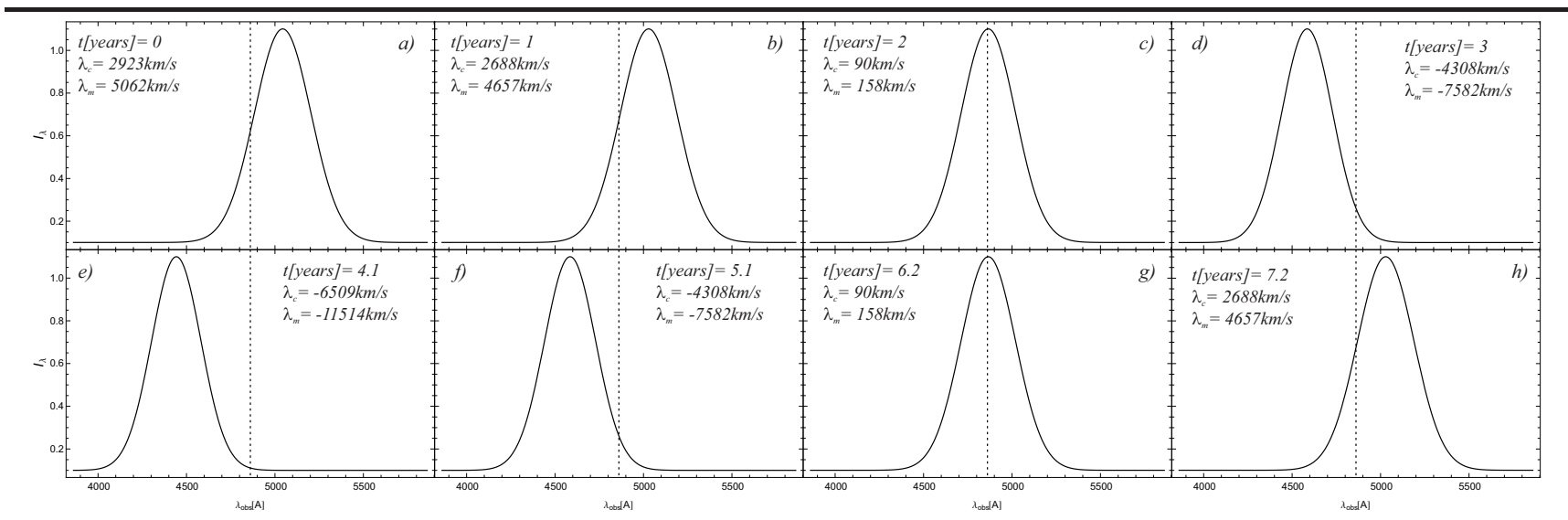

Fig. 6 The $\mathrm{H} \beta$ line for the case where only the lower mass BH in the binary system retains its emmiting BLR. Given parameter are same as in Fig. 3 but for single line.

the BLR radius, and finally the line velocity dispersion using the virial theorem. This model allows to explore time variability of the line parameters (shift, width and intensity) of a SMBBH system during an orbit, and can be applied for modeling of long term spectroscopic observations of the SMBBH candidates. We perform a number of simulations, changing the masses of components exploring how it's (not only the mass ratio that is usually is considered) affect the line profiles. Here we considered two cases: A) both BHs have a BLR, and B) only the smaller BH has a BLR From our investigation we can outline following conclusions:

In the case A)

(i) Beside the dynamical parameters and the mass ratio in a SMBBH system, the mass of components has an important role in the observed line profiles. It seems that low mass components (with total mass smaller than $10^{8} M_{\odot}$ ) will only moderately affect the broad line profiles. Consequently, the dynamic effect of binary system can be dominated by kinematical effects in BLRs and probably they are not good candidates for detection of SMBBHs using broad lines. The SMBBHs with bigger masses $\left(>10^{9} M_{\odot}\right)$ seems very promising for detection using SMBBHs broad lines. The line profiles show a complex shape, and line peak can be significantly shifted.

(ii) The line shapes are changing during an orbit of SMBBHs showing the double-peaked profile (two separated peaks) only in a short period in the case of massive black holes. This indicates that the double-peaked profiles may be not characteristic profiles observed from $\mathrm{SMBBH}$, i.e. one can expect to observe more frequently the asymmetric profiles from SMBBHs, and also some shoulders in the line profiles.

In the case B)

(iii) As it is well known, the emission of a single BLR in SMBBHs gives single shifted line, however, the shift and width of lines are larger in the case of more massive SMBBH components $\left(>10^{9} M_{\odot}\right)$ and they are easier to detect. During a period of revolution of binaries, one can expect that shift is changing form blue to red or vice versa, therefore the systematic shift to the blue or red in a period can indicate presence of a SMBBH.

Based on the dynamical time-scale of binary BH system we could say that the probability of detection of such an objects is rather moderate, since the the periods of the orbiting are in range of few years to few tens of the years. However, other parameters also has big influence, such are individual masses, mass ratio, inclinations, etc., which consequently lower the probability of detection binary system.

As a summary we can conclude that, beside dynamical effects, the total mass of a SMBBH has influence on the line shift and profile shape. Consequently, the observational effects in the broad lines seem to be present in massive systems $\left(>10^{9} M_{\odot}\right)$. However it is a question if the effects of a close binary system can be seen in low mass BHs, i.e. a large number of SMBBHs may give ordinary broad line profiles which are typical for a single AGN. Therefore, spectral monitoring of SMBBH candidates can be very useful for confirmation of their binary nature, i.e. the variation in the broad line profiles (shift, width and intensity) should be present, and this variation has to have a periodical or quasi-periodical nature following the orbiting period of a SMBBH.

\section{Acknowledgements}

This work is a part of the project (176001) "Astrophysical Spectroscopy of Extragalactic Objects," supported by the Ministry of Science and Technological Development of Serbia. 


\section{References}

Armitage, P. J. \& Natarajan, P. 2002, ApJL, 567, 9

Begelman, M. C., Blandford, R. D., Rees, M. J., 1980, Nature, 287, 307

Bogdanović, T. 2015, ASSP, 40, 103

Bon, E., Gavrilović, N., La Mura, G., Popović, L. Č. 2009, NewAR, 53, 121

Bon, E., Jovanovi, P., Marziani, P., Shapovalova, A.I., Bon, N., Borka Jovanovi, V., Borka, D., Sulentic, J., Popovi, L. ., 2012, ApJ, 759, 118

Boroson, T. A. \& Lauer, T. R. 2009, Nature, 458, 53

Cuadra, J., Armitage, P. J., Alexander, R. D. \& Begelman, M. C. 2009, MNRAS, 393, 1423

Decarli, R., Dotti, M., Montuori, C., T. Liimets, A. Ederoclite A., 2010a, ApJL, 720, L93

Eggleton, P. P., 1983, ApJ, 268, 368

Eracleous, M., Halpern, J. P. 1994, ApJS, 90, 1

Eracleous, M., Halpern, J. P., Gilbert, A. M., Newman, J. A., Filippenko, A. V., 1997, ApJ, 490, 216

Eracleous, M., Boroson, T. A., Halpern, J. P., \& Liu, J. 2012, ApJS, 201, 23

Gaskell, C. M., 1983, Liege International Astrophys. Colloq., 24, 473

Gaskell, C. M., 2010, Nature, 463, 1G

, Graham, M.J., Djorgovski, S. G., Stern, D., Glikman, E., Drake, A. J., Mahabal, A. A., Donalek, C., Larson, S. \& Christensen, E., 2015, Nature, 518, 74

Hayasaki, K., Mineshige, S. \& Sudou, H. 2007, PASJ, 59, 427

Kaspi, S.,Maoz, D., Netzer, H., Peterson, B. M., Vestergaard, M. \& Jannuzi, B. T., 2005, ApJ, 629, 61

Kaspi, S., Brandt, W. N., Maoz, D., Netzer, H., Schneider, D. P. \& Shemmer, O., 2007, ApJ, 659, 997

Kong, M. Z., Wu, X. B., Wang, R. \& Han, J. L., 2006, Chin. J. Astron. Astrophys, 6, 396

Komberg,B.V. 1968a, Ast. Zh., 45, 504

Komberg,B.V. 1968b, Sov. Ast., 12, 401

Komossa, S., Zhou, H., Lu, H. 2008, ApJL, 678, 81

Liu, X., Shen, Y., Bian, F., Loeb, A., Tremaine, S. 2014, ApJ, 789, 140L

Lodato, G., Nayakshin, S., King, A. R. \& Pringle, J. E. 2009, MNRAS, 398, 1392

Manmoto, T, 2010, ApJ, 534, 734

Narayan, R. \& Yi, I., 1994, ApJ, 428, L13

Marziani,P., Sulentic,J.W., Negrete,C.A., Dultzin,D., Zamfir,S., Bachev,R. 2010, MNRAS, 409, 1033

Marziani, P., Sulentic, J. W., Plauchu-Frayn, I., del Olmo, A. 2013, A\&A, 555A, 89

Paczynski, B., 1971, ARA\&A, 9, 183

Peterson, B. 2014, SSRv, 183, 253

Popović, L. Č. 2012, NewAR, 56, 74

Popović, L. Č., Mediavilla, E., Bon, E. \& Ilić, D. 2004, A\&A, 423, 909

Popović, L. Č., Mediavilla, E. G., Pavlović, R. 2000, SerAJ, 162, 1

Popović, L. Č., Shapovalova, A. I., Ilić, D., Kovačević, A., Kollatschny, W., Burenkov, A. N., Chavushyan, V. H., Bochkarev, N. G., Leon-Tavares, J. 2011, A\&A, 528A, 130
Robinson, A., Young, S., Axon, D. J., Kharb, P. \& Smith, J. E., 2010, ApJL, 717, 122

Runnoe, J.C., Eracleous, M., Mathes, G. et al., 2015, ApJS, 221, 7R

Shakura, N.I., \& Sunyaev, R. A., 1973, A\&A, 24, 337

Shapovalova, A.I., Popović, L. Č., Chavushian, V. et al. 2015, sent to ApJSS - under review

Shen, Y., \& Loeb, A. 2010, ApJ, 725, 249

Strateva,I.V., Strauss,M.A., Hao,L. 2003, AJ, 126, 1720

Sulentic,J.W., Marziani,P., Zamfir,S. 2009 NewAR, 53, 198S

Sulentic, J. W., Marziani, P. Dultzin-Hacyan, D., 2000, ARAA 38, 521

Tsalmantza, P., Decarli, R., Dotti, M., \& Hogg, D. W. 2011, ApJ, 738, 20

Woo, J. H., Cho, H., Husemann, B., Komossa, S., Bennert, V. \& Park, D., 2013, AAS, 222, 309.04W

Woo, J. H., Cho, H., Husemann, B., Komossa, S., Park, D. \& Bennert, V. N., 2014, MNRAS, 437, 32W

Wu, X. B., Wang, R., Kong, M. Z., Liu, F. K. \& Han, J. L., 2004, A\&A, 424, 793

Yan, C. S., Lu, Y., Yu, Q., Mao, S., Wambsganss, J., 2014, ApJ, 784, 2

Yan, C. S., Lu, Y., Dai, X. \& Yu, Q., 2015, ApJ, 809, 117

Yu, Q., \& Lu, Y., 2001, A\&A, 377, 17

Zamfir, S., Sulentic, J. W., Marziani, P. and Dultzin, D., 2010, MNRAS, 403, 1759

This manuscript was prepared with the AAS LATEX macros v5.2. 